\title{
A Novel Scheme to Detect Optical DPSK Signals
}

\author{
E. Ciaramella, G. Contestabile, and A. D'Errico
}

\begin{abstract}
We propose and demonstrate a novel approach to detect optical differential phase-shift keying signals. The technique is based on differential phase-to-polarization conversion in a polarization-maintaining fiber, so that the polarization-modulated signal can be detected by using a polarizer and a common intensity modulation receiver.
\end{abstract}

Index Terms-Optical fiber communication, optical modulation formats, polarization-maintaining fiber (PMF).

\section{INTRODUCTION}

I N OPTICAL transmission systems, the intensity modulation (IM) is used, mostly with nonreturn-to-zero (NRZ) format, but sometimes with the return-to-zero (RZ) format. However, for next-generation systems, it has been proposed that differential phase-shift keying (DPSK) may be preferred because of its high robustness to nonlinear propagation [1] and, to a smaller extent, to polarization-mode dispersion (PMD) [2]. Although these features could pave the way to a new design of transmission systems (e.g., with higher transmission power and quite longer unrepeatered span), the IM can still be preferred because of the simplicity of the receiver.

Up to now, three main solutions for detecting optical DPSK are known. The first is based on a well-known scheme directly taken from coherent communications, which requires a laser field used as a local oscillator. As this has to match both the state of polarization (SOP) and the carrier frequency of the signal [3], [4], this leads to complex and expensive receiver design.

Another scheme is based on a Mach-Zehnder interferometer with one bit delay in one arm [5]; this scheme can be used to convert the DPSK signal to an IM signal, which is then received by means of a usual receiver. The delay interferometer can be implemented as an integrated component using planar waveguide technology [6]. However, as all interferometric structures, it might be difficult to stabilize, as it can critically suffer from environmental fluctuations and strongly depend on the bias stability.

A third scheme exploits proper injection locking of a semiconductor laser to convert a DPSK signal into an intensity modulated signal [7].

Here we present a novel scheme that can be used to detect optical DPSK signals using established and low-cost components [8]. We first convert a DPSK signal into a polarization shift-keying (POLSK) signal. This is accomplished by means

Manuscript received March 23, 2004; revised May 13, 2004. This work was supported in part by Marconi Communications SpA under a grant.

E. Ciaramella and G. Contestabile are with Scuola Superiore Sant'Anna, 56124 Pisa, Italy, and also with CNIT, Photonic Networks National Laboratory, 56124 Pisa, Italy (e-mail: ernesto.ciaramella@cnit.it).

A. D'Errico is with CNIT, Photonic Networks National Laboratory, 56124 Pisa, Italy.

Digital Object Identifier 10.1109/LPT.2004.831961 of a birefringent element, e.g., a polarization-maintaining fiber (PMF). From a theoretical point of view, this is similar to a Mach-Zehnder delay interferometer, but it produces a POLSK signal at the output. The POLSK signal is then converted to IM by means of a polarization beam splitter (PBS). Depending on the amount of birefringence, we can either produce an NRZ-like (as for delay interferometers) or RZ signal.

\section{OPERATING PRINCIPLE}

As it is well known, two orthogonal principal states of polarization (PSPs) exist in a PMF which define two linearly SOPs that propagate with no significant distortion, but with a fixed differential group delay (DGD) along the PMF.

In the following, we will show how this effect can be used to implement DPSK detection. Let us suppose that the input signal is an ideal undistorted DPSK signal that can be written as

$$
\vec{E}(t)=\left(E_{0, x} \vec{x}+E_{0, y} \vec{y}\right) e^{i \Phi(t)}
$$

where $\vec{x}$ and $\vec{y}$ versor indicate the two PSPs of the PMF and the phase is

$$
\Phi(t)=\pi \sum_{k} a_{k} q\left(t-k T_{\mathrm{bit}}\right)
$$

(here $a_{k}=0,1, q(x)=1$, for $|x|<T_{\mathrm{bit}} / 2$, and $q(x)=0$, for $|x| \geq T_{\text {bit }} / 2$ ).

If the input signal SOP is set to be linearly polarized at $45^{\circ}$ with respect to the PSPs, it splits into two identical components with orthogonal linear polarization, each parallel to one of the main axes of the PMF. In the PMF, these actually propagate with different group and phase velocity. At the PMF output, the two signal components have acquired a significant relative group delay, and also an optical-phase difference

$$
\vec{E}(t)=E_{0, x} e^{i \Phi\left(t-T+\frac{\Delta T}{2}\right)+\frac{i \Psi}{2}} \vec{x}+E_{0, y} e^{i \Phi\left(t-T-\frac{\Delta T}{2}\right)-\frac{i \Psi}{2}} \vec{y}
$$

where $T$ is the average propagation time, $\Delta T$ is the DGD, and $\Psi$ is the phase difference. Let us neglect the phase $\Psi$, as it affects the polarization in such a way that can be fully compensated for by means of a common PC.

If we choose $\Delta T=T_{\mathrm{bit}}$, it can be seen that the input phase modulation turns out into a polarization modulation. In our case, two orthogonal SOPs can be produced, depending on the logic XOR of two subsequent DPSK symbols. Indeed, the final, normalized output Jones vector is given by

$E_{\text {out }}(t)=\frac{1}{\sqrt{2}}\left(\begin{array}{c}e^{i \Phi\left(t-T+\frac{\Delta T}{2}\right)} \\ e^{i \Phi\left(t-T-\frac{\Delta T}{2}\right)}\end{array}\right)=\frac{e^{i \Phi\left(t^{\prime}\right)}}{\sqrt{2}}\left(\begin{array}{c}1 \\ e^{i\left(\Phi\left(t^{\prime}-\Delta T\right)-\Phi\left(t^{\prime}\right)\right)}\end{array}\right)$

where $t^{\prime}=t-T+\Delta T / 2$, and the common $\Phi\left(t^{\prime}\right)$ phase is unessential. Hence, the phase difference between two subse- 


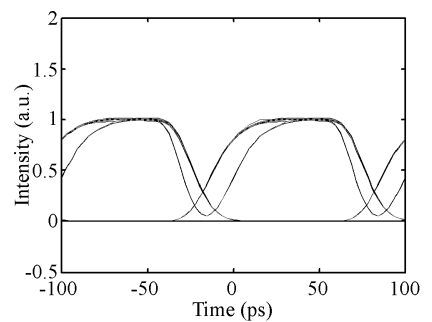

(a)

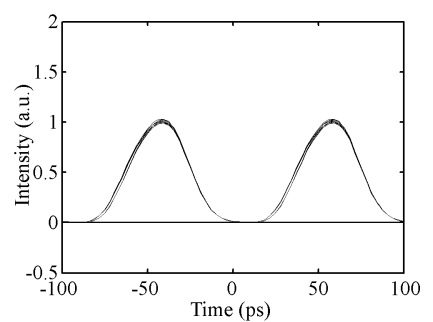

(b)
Fig. 1. 10-Gb/s eye diagrams obtained by simulating the receiver behavior and using a fiber (a) DGD $=100$ and (b) 50 ps.

quent bits may be either zero or $\pm \pi$. These two cases correspond, respectively, to the output SOP linearly polarized either at $45^{\circ}$ or at $-45^{\circ}$. A polarizing element, such as a polarizer or a PBS, can then be used to convert the output signal into an IM NRZ signal: If this is set at $-45^{\circ}$, the final output intensity is

$$
I_{\mathrm{pol}}(t)=I_{0} \sin ^{2}\left(\frac{\Phi\left(t^{\prime}-\Delta T\right)-\Phi\left(t^{\prime}\right)}{2}\right) .
$$

We note that this is the same expression we would obtain for an interferometer with $\Delta T$ delay. From (5), it can also be derived that, in the same scheme, if the fiber $\Delta T$ is significantly lower than the bit time, an RZ IM signal can be produced.

Furthermore, when using a PBS, the two output ports can be used to achieve the balanced detection and its 3-dB sensitivity improvement, in a similar way as for the integrated delay interferometers.

We first use numerical simulations to check this scheme. We assume a 10-Gb/s DPSK signal [produced by means of an $8-\mathrm{GHz}$ bandwidth phase modulator (PM)], injected at $45^{\circ}$ with respect to the PMF PSPs. The DGD is either 100 or 50 ps, and at the PMF output a polarization controller (PC) and a polarizer are used. The obtained eye diagrams (filtered with a 30-GHz low-pass filter) are reported in Fig. 1. Note that a quasi-NRZ signal is produced [Fig. 1(a)] when DGD $=100 \mathrm{ps,}$ showing a notch between two subsequent marks, which indeed is similar to what is obtained using the delay interferometer [5]. On the other hand, if DGD $=50 \mathrm{ps,} \mathrm{we} \mathrm{obtain} \mathrm{an} \mathrm{RZ} \mathrm{signal,}$ as it is shown in Fig. 1(b). Simulations confirm that the two PBS output signals can be also successfully used in a balanced detection scheme.

\section{EXPERIMENT}

To assess the effectiveness of the proposed scheme, we used the experimental setup that is shown in Fig. 2. A $\mathrm{LiNbO}_{3} \mathrm{PM}$ is used to produce a $9.952-\mathrm{Gb} / \mathrm{s}$ (STM-64 or OC 192) DPSK signal. As we use a pseudorandom bit sequence (PRBS) signal $\left(2^{31}-1\right.$ bit $)$, we do not need the differential encoder [6]. At the receiver side, two PMFs can be used, with DGD $=95$ or 50 ps, respectively. A fiber $\mathrm{PC}$ is used to have the input signal with linear polarization at $45^{\circ}$; another $\mathrm{PC}$ is used to properly rotate the polarization with respect to the PBS axis. After the PBS, the output IM signal is detected by means of a common receiver or a fast photodiode. Note that temperature variations may induce

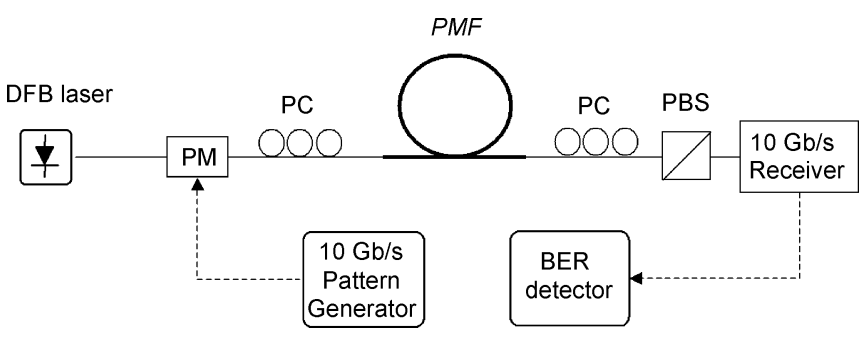

Fig. 2. Experimental setup.

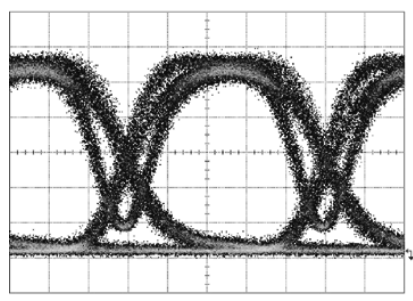

(a)

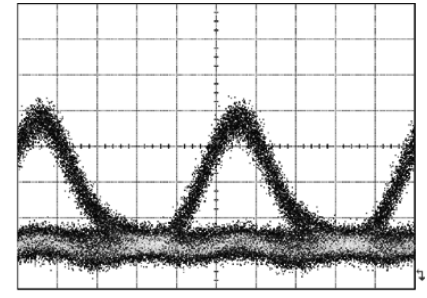

(b)
Fig. 3. 10-Gb/s eye diagrams after the PBS for a PMF with (a) DGD $=95 \mathrm{ps}$ and (b) DGD $=50 \mathrm{ps}$. In both cases, the time scale is $20 \mathrm{ps} / \mathrm{div}$.

SOP changes at the input and at the output of the PMF. Hence, for a practical application, the two PCs should be dynamically optimized (e.g., using the commercially available endless PCs).

In Fig. 3, we report the corresponding eye diagrams taken with a fast photodiode (40-GHz bandwidth). As mentioned above, if the DGD is close to the bit rate, we observe a quasi-NRZ eye diagram (Fig. 3(a), taken for DGD $=95 \mathrm{ps)}$ ). When the DGD is much lower, the produced signal tends to resemble an RZ eye diagram [see Fig. 3(b)], a feature that can also be observed with delay interferometers. The agreement with simulation results shown in Fig. 1 is apparent.

We then performed bit-error-rate (BER) measurements. As in [6], we take advantage of the PRBS property to avoid any electronic differential encoder (the coded sequence is the original one, shifted by some bits).

A common SDH STM-64 $(10 \mathrm{~Gb} / \mathrm{s})$ receiver was now used. By setting a variable optical attenuator, we measured the BER value as a function of the optical power at the receiver input. A similar curve was obtained for a common IM 10-Gb/s NRZ signal. The obtained results are shown in Fig. 4, where we report the data measured for IM (dots), and for DPSK using a PMF with DGD $=95$ or 50 ps (triangles and circles, respectively). As can be seen, the three curves are quite similar. The quasi-NRZ signal suffers from a slight penalty (less than $0.3 \mathrm{~dB}$ ). This is likely due to the unideal balance of the demodulating part (which was manually set). This slight difference in performance might also be attributed to the receiver, optimized for an NRZ signal. These results confirm the validity of the proposed scheme. We tested our technique in a transmission experiment over $2 \times 80 \mathrm{~km}$ nonzero dispersion fiber (NZDF) with inline amplifiers and dispersion compensation; in that case, we found no relevant penalty (around $0.5 \mathrm{~dB}$ ). The signal SOP variations were found to change dynamically, over 1-min time scale (so that manual control of the PC could be used). 


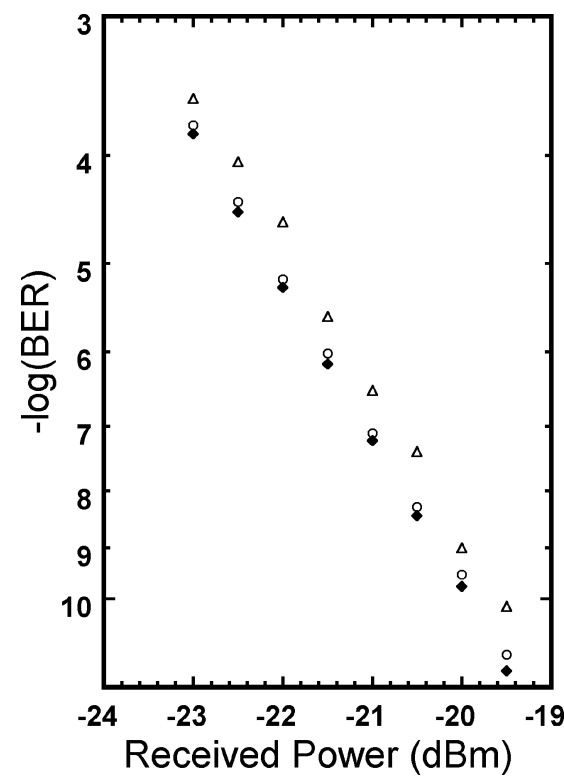

Fig. 4. 10-Gb/s BER curves for IM direct detection NRZ (black dots), DPSK converted to quasi-NRZ (triangles) DPSK converted to IM-RZ (circles).

\section{CONCLUSION}

We have presented and demonstrated a simple and practical scheme to detect an optical DPSK signal. The scheme makes use of a common PMF to convert the differential phase modulation into a polarization modulation, which can be detected by means of a polarizer. BER measurements confirm the good performance of the proposed receiver.

We would like to point out that most of this scheme can be also viewed as a one-stage PMD compensator [9]. It might be possible that, in presence of a PMD-affected link, this receiver could take advantage of this intrinsic functionality. As an example, the highest PMD impairments usually arise when the fiber link has a quite high DGD and the signal is equally split between the PSPs [9]. In that case, if the PSPs of the PMF are aligned to those of the line, the combined first-order effect would be similar to that of a single piece of PMF having the sum (or difference) of the two DGDs (which should be minimized). Hence, in a given range of fiber DGD, the whole receiver might produce just pulsewidth variations on behalf of distortions (depending on the DGD values of the fiber and PMF). To this aim, the PMF DGD should be carefully chosen and a properly designed control and feedback for the PC would have to be introduced.

Finally, in the above discussion, we have assumed a PMF as the birefringent medium. It is worth noting that for practical applications other solutions may also be considered, e.g., a birefringent crystal [10].

\section{REFERENCES}

[1] M. Rohde, C. Caspar, N. Heimes, M. Konitzer, E.-J. Bachus, and N. Hanik, "Robustness of DPSK direct detection transmission format in standard fiber WDM systems," Electron. Lett., vol. 36, pp. 1483-1484, 2000.

[2] C. Xie, L. Möller, H. Haunstein, and S. Hunsche, "Comparison of system tolerance to polarization mode dispersion between different modulation formats," IEEE Photon. Technol. Lett., vol. 15, pp. 1168-1170, Aug. 2003.

[3] T. Chikama, S. Watanabe, T. Naito, H. Onaka, T. Kyonaga, Y. Onoda, H. Miyata, M. Suyama, M. Seino, and H. Kuwahara, "Modulation and demodulation techniques in optical heterodyne PSK transmission system," J. Lightwave Technol., vol. 8, pp. 309-322, Mar. 1990.

[4] B. Glance, "Polarization independent coherent optical receiver," $J$. Lightwave Technol., vol. LT-5, pp. 274-276, Feb. 1987.

[5] E. Swanson, J. Livas, and R. Bondurant, "High sensitivity optically preamplified direct detection DPSK receiver with active delay line stabilization," IEEE Photon. Technol. Lett., vol. 6, pp. 263-265, Feb. 1994.

[6] A. H. Gnauck, S. Chandrasekhar, J. Leuthold, and L. Stulz, "Demonstration of 42.7-Gb/s DPSK receiver with 45 photons/bit sensitivity,' IEEE Photon. Technol. Lett., vol. 15, pp. 99-101, Jan. 2003.

[7] Y. Awaji, T. Kuri, M. Naganuma, K. Kitayama, and W. Chujo, "Differential-phase-to-intensity conversion (DPIC) based on injection locking of a semiconductor laser," Opt. Lett., vol. 26, no. 20, pp. 1538-1540, 2001.

[8] "Technique for converting the modulation format of an optical signal," patent pending.

[9] H. Sunnerud, M. Karlsson, C. Xie, and P. A. Andrekson, "Polarization mode dispersion in high speed fiber-optic transmission systems," J. Lightwave Technol., vol. 20, pp. 2204-2219, Dec. 2002.

[10] J. N. Damask, P. R. Myers, A. Boschi, and G. J. Simer, "Demonstration of a coherent PMD source," IEEE Photon. Technol. Lett., vol. 15, pp. 1612-1614, Nov. 2003. 